\title{
High-energy THz pulses for electron acceleration
}

\author{
Franz X. Kärtner \\ Center for Free-Electron Laser Science and Deutsches Elektronen-Synchrotron (DESY), Notkestraße 85, 22607 Hamburg, Germany \\ Department of Physics and Center for Ultrafast Imaging, University of Hamburg, Luruper Chaussee 149, 22761 Hamburg, Germany \\ e-mail address: franz.kaertner@desy.de
}

\begin{abstract}
Recent theoretical and experimental results on laser based high-energy single-cycle and multi-cycle terahertz pulse generation are discussed. The pulse formats are chosen to demonstrate various $\mathrm{THz}$ accelerating and beam manipulation devices. Results for a segmented terahertz electron manipulator and accelerator are presented.

OCIS codes: (320.0320) Ultrafast Optics; (190.4410) Nonlinear optics, parametric processes; (190.7110) Ultrafast nonlinear optics; (160.3730) Lithium Niobate; (320.7090) Ultrafast lasers.
\end{abstract}

\section{Introduction}

The availabiltiy of high power and high energy Terahertz $(\mathrm{THz})$ radition opens up the possibility to use it for particle acceleration. Today, high brightness and highly relativistic electron beams are generated by circular or linear accelerators (LINAC) typically operating at a few $\mathrm{GHz}$ accelerating frequencies and approaches towards X-band frequencies in the $10 \mathrm{GHz}$ range are maturing. The achievable accelerating gradients are limited by field emission from cavity walls influenced by pulsed heating to several tens of $\mathrm{MV} / \mathrm{m}$ in the case of low frequencies and up to 100 $\mathrm{MV} / \mathrm{m}$ in the case of X-band frequencies. Short electron bunches are typically created by photoemission from the cathode in the presence of strong accelerating fields followed by bunch compression. Low charge bunches, 1-10 pC, may be compressed to durations down to $10-30$ fs in duration. For a given accelerating field strength and RF frequency compression is limited by space charge. With higher operating frequency, higher gradients in the accelerating electric field arise which enhances velocity bunching and leads to the generation of shorter electron bunches. These short electron bunches can be used for ultrafast electron diffraction or intense X-ray production. Choosing an operating frequency of the accelerator in the THz range, i.e. here $0.1-0.5 \mathrm{THz}$ and using multi-cycle to single-cycle pulses, accelerating fields in the 0.3 to $1 \mathrm{GV} / \mathrm{m}$ range are sustainable and bunch compression to the sub-femtosecond level of significant charge on the order of $\sim 1 \mathrm{pC}$ becomes possible. However, to drive electron guns and accelerators high-energy single-cycle and multi-cylce $\mathrm{THz}$ pulses in the $\mathrm{mJ}$ and tens of $\mathrm{mJ}$ range, respectively, are necessary. Here, we discuss approaches towards this goal and results achieved sofar.

\section{High energy THz pulse generation}

The traditional approach towards generation of single-cycle $\mathrm{THz}$ pulses is via optical rectification in Lithium Niobate using the tilted pulse front technique (TPFT) [1]. Using 1 micron, sub-ps pulses up to $0.4 \mathrm{~mJ} \mathrm{THz}$ pulses have been generated with up to $0.8 \%$ efficiency albeit the center frequency moved below $0.2 \mathrm{THz}$, which would then need even higher $\mathrm{THz}$ energies as a driver, since corresponding structures are larger [2]. Here, we report ont the generation of single-cycle $\mathrm{THz}$ pulses with a center frequency of $0.28 \mathrm{THz}$, an energy of $0.2 \mathrm{~mJ}$ and a conversion efficiency of $0.5 \%$ using $40-\mathrm{mJ}, 1020-\mathrm{nm}, 1-\mathrm{ps}$ pulses from a cryogenic Yb:YLF laser generated with the TPFT method in cryogenically cooled Lithium Niobate. Numerical simulations show, that further scaling to $1 \%$ efficiency with $100 \mathrm{~mJ}$ driver pulses is possible and should result in $1 \mathrm{~mJ} \mathrm{THz}$ pulses at about $0.3 \mathrm{THz}$ wavelength. Cryogenic cooling of Lithium Niobate lowers the absorption at $\mathrm{THz}$ frequencies. The conversion efficiency is limited due to break-up of the driver pulses after excessive cascading [3].

For multi-cycle or narrowband $\mathrm{THz}$ generation, difference frequency generation (DFG) in cryogenically cooled periodically poled lithium niobate (PPLN) crystals using quasi-phase matching promises high conversion efficiency of several percent. Cryogenic cooling allows the use of long crystals and cascading does not lead to break-up of the driver pulses as in the TPFT case due to collinear interaction of the waves [4]. DFG using highenergy broadband Ti:Sappire pulses and the chirp and delay method to match the difference frequency to the quasiphase matching period resulted in $40 \mu \mathrm{J}$ pulses at $0.5 \mathrm{THz}$ and $260 \mathrm{ps}$ duration [5]. The use of large aperture crystals fabricated by the Taira group and linearization of the difference frequency between the chirped and delayed pulses lead to further improvements by more than an order of magnitude to produce $460 \mu \mathrm{J}$ of $\mathrm{THz}$ radiation with about $460 \mathrm{~mJ}$ of laser pulses [6]. The future goal is to generate with $5 \%$ conversion efficiency up to $50 \mathrm{~mJ}$ of multicylce $\mathrm{THz}$ pulses from 1-J laser pulses to drive a THz accelerator using dielectrically loaded metal waveguides [7]. 


\section{THz acceleration}

A proof of principle $\mathrm{THz}$ acceleration experiment based on laser-generated single-cycle $\mathrm{THz}$ pulses with $10 \mu \mathrm{J}$ pulse energy at $0.45 \mathrm{THz}$ has been performed recently [8]. First THz guns based on a parallel plate $\mathrm{THz}$ waveguide have been reported with up to $400 \mathrm{eV}$ mean energy electron bunches and 5\% energy spread [9]. More advanced THz gun structures driven by single-cycle $\mathrm{THz}$ pulses have been proposed [10]. The single-cycle gun structure is a segmented $\mathrm{THz}$ waveguide device composed of arrays of parallel plate waveguides with dielectric fillings to delay
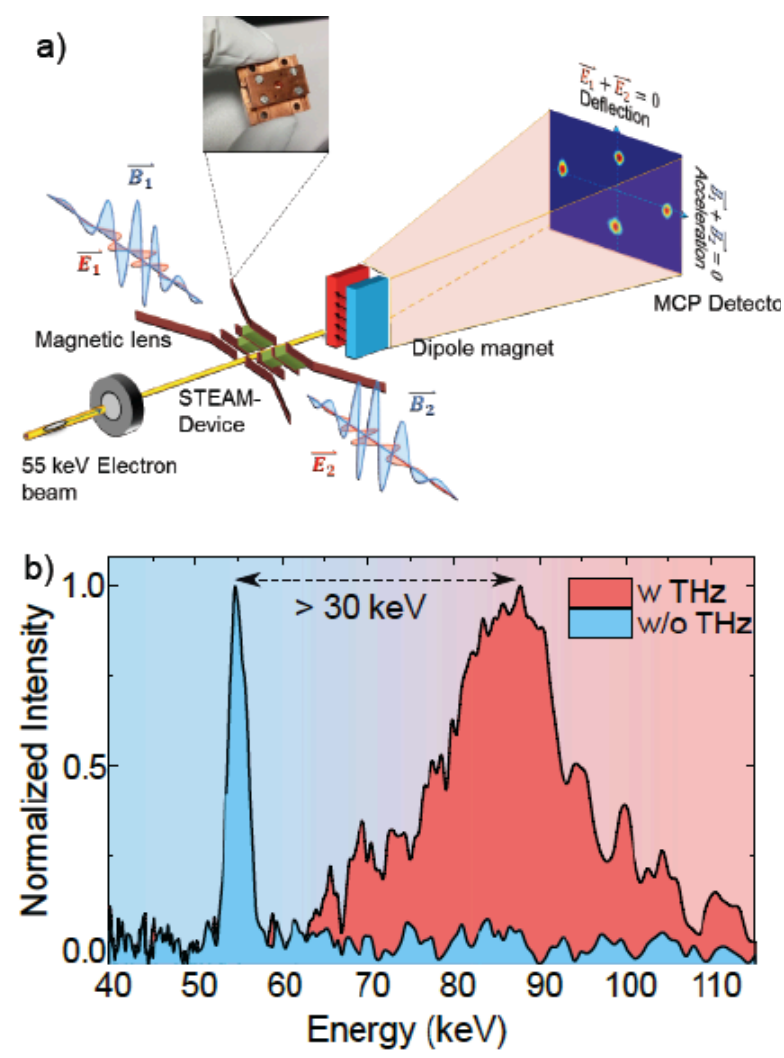

Fig. 1: (a) Acceleration of an electron bunch from a $55 \mathrm{keV}$ DC-gun using a segmented $\mathrm{THz}$ waveguide acceleration device with dipole magnet for energy measurement on MCP; (b) electron energy spectrum without (black) and with $\mathrm{THz}$ pulse(red). [11] the $\mathrm{THz}$ pulses in each waveguide, such that when excited with a single high energy $\mathrm{THz}$ pulse jointly, a tilted pulse front is created at the interaction point with electrons. This leads to continuous acceleration of the electron bunch in the interaction areas, see Fig. 1 (a). To demonstrate this single cycle accelerator concept, we used a photo- triggered, 55 $\mathrm{kV}$ DC-gun to inject an electron bunch into the accelerating structure. The accelerating structure is driven by two single-cycle $\mathrm{THz}$ pulses with about 10 $\mu \mathrm{J}$ of $\mathrm{THz}$ energy. Fig. 1 (b) shows the energy spectrum of the accelerated electron bunch, measured by a dipole magnet and an MCP. The spectrum measured with and without the $\mathrm{THz}$ pulses are shown. The $\mathrm{THz}$ accelerator boosts the average electron energy by $35 \mathrm{keV}$ and significantly broadens the energy distribution due to the long electron bunch from the DC-gun of about $500 \mathrm{fs}$, filling a significant portion of the accelerating $\mathrm{THz}$ field cylce. For acceleration, the two $\mathrm{THz}$ pulses are injected such that the electric fields constructively interfere in the center of the structure, and, in forward direction. The transvers magnetic fields are canceled. If the pulses are delayed with respect to each other by half a $\mathrm{THz}$ wavelength, the electric fields cancel in the center of the device and the transvers magnetic fields add, which leads to a transvers deflection of the electron bunch. This operation mode can be used for streaking of the electron bunch to measure its duration [11]. The deflection strength allows for sub-10 femtosecond resolution. The structure has been also used to demonstrate temporal pulse compression, focussing and defocussing.

Currrent work is geared towards pushing laser based $\mathrm{THz}$ generation to the multi-mJ level using Joule level, picosecond laser pulses with optimized pulse formats [4]. Such high energy THz pulses can accelerate electrons to the 10-20 MeV level, energetic enough to enable in a first hard X-ray source via inverse Compton scattering [12].

\section{References}

[1] J. Hebling, G. Almasi, I. Z. Kozma, J. Kuhl, Opt. Exp. 10, 1161 (2012).

[2] J. A. Fülop, Z. Ollmann, C. Lombosi, C. Skrobol, S. P. Klingebiel, L. Palfalvi, F. Krausz, S. Karsch, J. Hebling, Opt. Exp. 22 , 20155 (2014).

[3] K. Ravi, W. R. Huang, S. Carbajo, X. Wu and F. X. Kärtner, Opt. Exp. 22, 20239 (2014).

[4] K. Ravi, et al. Opt. Lett. 41, 3806 (2016) and Opt. Lett. 42, pp. 25582 (2016).

[5] F. Ahr, S. W. Jolly, N. H. Matlis, S. Carbajo. T. Kroh, K. Ravi, D. N. Schimpf, J. Schulte, H. Ishizuki, T. Taira, A. Maier and F. X. Kärtner, Opt. Lett.

[6] S. W. Jolly, F. Ahr, N. H. Matlis, V, Leroux, T, Eichner, K. Ravi, H. Ishizuki, T. Taira, F. X. Kärtner abd A. R. Maier, this conference.

[7] L. J. Wong, A. Fallahi and F. X. Kärtner, Opt. Express 21, 9792 (2013).

[8] E. A. Nanni, W. R. Huang, K. Ravi, A. Fallahi, G. Moriena, R. J. Miller, and F. X. Kärtner, Nat. Communications 6, p. 8486 (2015).

[9] W. R. Huang, et al, Optica 3, p. 1209 (2016).

[10] A. Fallahi, M. Fakhari, A. Yahaghi, M. Arrieta, and F. X. Kärtner, Phys. Rev. Accelerators and Beams 19, 081302 (2016).

[11] D. Zhang, A. Fallahi, M. Hemmer, X. Wu, M. Fakhari, Y. Hua, H. Cankaya, A.-L. Calendron, L. Zapata, N. H. Matlis, and F. X. Kärtner, Nat. Photonics doi.org/10.1038/s41566-018-0138-z

[12] F. X. Kärtner et al. doi:10.1016/j.nima.2016.02.080. 